\title{
Profesionalizando el patrimonio cultural mexicano: el caso de la Maestría en Patrimonio Cultural de México del instituto de artes de la UAEH
}

Professionalizing the Mexican cultural heritage: the case of the master in cultural heritage of México from the School of Arts of the UAEH

Carmen Lorenzo-Monterrubio ${ }^{a}$, Arturo Vergara-Hernández ${ }^{b}$, María Esther Pacheco-Medina ${ }^{c}$

\begin{abstract}
:
The Cultural Heritage is a subject that today is discussed in educational and cultural institutions, in public and private organizations and in associations or civil groups. The growing interest in the Cultural Heritage of Mexico contributed to the creation of a postgraduate program, the first offered at the School of Arts of the Universidad Autónoma del Estado de Hidalgo.
\end{abstract}

Keywords:

Cultural heritage of Mexico, professionalization, Master in cultural heritage of Mexico

\section{Resumen:}

El Patrimonio Cultural es un tema que hoy se discute en instituciones educativas y culturales, en organismos públicos y privados y en asociaciones o agrupaciones civiles. El creciente interés por el Patrimonio Cultural de México contribuyó a la creación de un programa de posgrado, el primero que se oferta en el Instituto de Artes de la Universidad Autónoma del Estado de Hidalgo.

\section{Palabras Clave:}

Patrimonio cultural de México, profesionalización, Maestría en patrimonio cultural de México

\footnotetext{
a Autor de Correspondencia, Universidad Autónoma del Estado de Hidalgo, Instituto de Artes, ORCID: 0000-0002-0906-0531, Email: alorenzo@uaeh.edu.mx

${ }^{\mathrm{b}}$ Universidad Autónoma del Estado de Hidalgo, Instituto de Artes, ORCID: 0000-0002-4756-6381, Email: vergarah@uaeh.edu.mx c Universidad Autónoma del Estado de Hidalgo, Instituto de Artes, ORCID: 0000-0001-7540-6249, Email: maria_pacheco6925@uaeh.edu.mx
} 


\section{Introducción}

México posee un gran Patrimonio Cultural. Éste se puede definir como el acervo de los bienes de la sociedad (que pueden ser tangibles o intangibles), de origen propio o ajeno, que son necesarios para la reproducción social y cultural del pueblo que los sustenta como suyos.

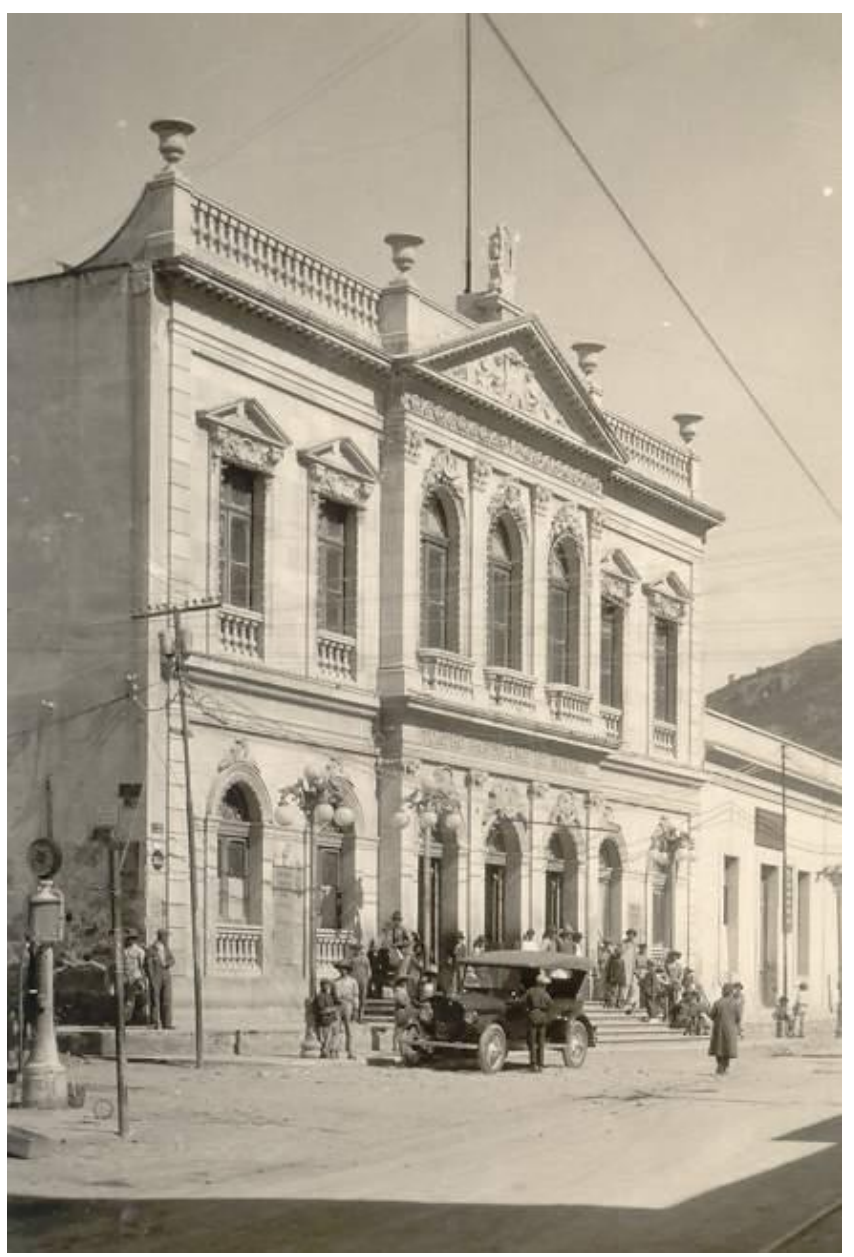

Figura 1. Ejemplo de patrimonio perdido: el Teatro Bartolomé de Medina, derribado en 1943. cronistadehidalgo.com.mx

El Patrimonio Cultural de nuestro país es producto de su larga historia y de la diversidad étnica que lo caracteriza. En el devenir del tiempo, cada vez son más las personas interesadas en el conocimiento, manejo, protección y aprovechamiento del patrimonio cultural.

A través de sus instituciones, el Estado Mexicano se ha encargado de este aspecto fundamental del país. El Instituto Nacional de Antropología e Historia y la Escuela Nacional de Antropología e Historia (dependiente del primero), han sido los principales encargados de proteger por un lado y preparar por el otro, a quienes se dedican a la investigación, promoción y difusión del Patrimonio Cultural mexicano. Pero la demanda cada vez mayor de profesionales de este tema al interior del país, dio pie a que la Universidad Autónoma del Estado de Hidalgo analizara la viabilidad y pertinencia de un programa de posgrado de este tipo, y aperturara en 2018, la primera maestría de su Instituto de Artes dedicada a la profesionalización de quienes que están o estarán laborando en los organismos y organizaciones civiles dedicadas a la gestión del arte y el patrimonio cultural.

Así, la Maestría en Patrimonio Cultural de México (MPCM) es el primer programa de posgrado que oferta el Instituto de Artes de la Universidad Autónoma del Estado de Hidalgo, a 16 años de su creación. Constituye un programa novedoso y de actualidad, ya que hoy por hoy se requiere comprender el concepto de Patrimonio Cultural para su mejor aprovechamiento, y para promover la identidad de los pueblos, fortaleciendo su sentido de pertenencia. Al conjugar arte y cultura, con un sentido multidisciplinar, se busca ofrecer medidas para la protección y la conservación de bienes culturales, materiales e inmateriales, que resultan de enorme fragilidad y que ante el avance avasallador de la modernidad están en riesgo constante. No debemos perder de vista que de acuerdo con Enrique Florescano (1993), el Patrimonio Cultural es un constructo social y por lo tanto, es necesario incorporar a las propias comunidades culturales a las grandes y pequeñas decisiones sobre el particular, no dejando esto sólo en manos del Estado.

\section{Antecedentes}

El de Artes es el más joven de los institutos de la UAEH. Fue creado en el año 2002 con tres licenciaturas: Artes Visuales, Música y Danza, y un año después se incorporó Arte Dramático. Después de una década de contar con cuatro programas académicos de licenciatura, se buscó que el Instituto de Artes tuviera un primer programa de posgrado; por ser un instituto dedicado a las artes se pensó en un inicio que podría ser una Maestría en Historia del Arte, debido a que esta disciplina está siempre en la currícula de las áreas artísticas de nivel licenciatura.

Fue hasta el año 2011 cuando se realizaron de manera formal los estudios de pertinencia y factibilidad de esta propuesta educativa, ya que como se mencionó, el estudio del arte con un enfoque histórico es un denominador común de los cuatro programas de licenciatura (Artes Visuales, Música, Danza y Teatro). Estos estudios se particularizaron en los temas de Necesidades Sociales, Comparativo de planes y programas educativos, Demanda educativa potencial, 
Mercado laboral, Disciplina y la Profesión; así como Recursos del posgrado; fue elaborado por la Mtra. Dulce Olivia Fosado Martínez y la Dra. Silvia Nicolás Cisneros (2011).

Entre los años 2013 a 2015 se trabajó entonces en el diseño curricular de la Maestría en Historia del Arte, logrando tener un $95 \%$ de avance aproximadamente, pero con el cambio de Dirección del Instituto que pasó del Lic. Juan Randell Badillo al Lic. Gonzalo Villegas de la Concha en 2015, se identificó la necesidad de actualizar los estudios de pertinencia y factibilidad, ya que los realizados en 2011, después de cinco años, resultaron anacrónicos.

Después de analizar la experiencia en investigación y docencia de los profesores que conformarían el núcleo académico básico del nuevo programa de posgrado, en 2016 se realizaron nuevos estudios de pertinencia y factibilidad de la Maestría en Patrimonio Cultural, pues se identificó que este tema era viable y con demanda, representando también un interés particular de alumnos y profesores del Instituto. Así mismo, el interés creciente sobre temas del patrimonio cultural por parte de la población (especialmente la que se encontraba laborando ya en este ámbito disciplinar pero sin la debida profesionalización), se vio reflejado en la orientación de dichos estudios, por lo que se procedió a entrevistar no solo a la comunidad del Instituto de Artes, sino también a personal y alumnos de otras instituciones educativas y culturales. En un principio se pensó enfocar el nuevo programa hacia el patrimonio cultural del estado de Hidalgo pero posteriormente se valoró la necesidad de extenderse a todo el país, pues se consideró que no era correcto descontextualizar el patrimonio cultural hidalguense del ámbito nacional, incluso internacional. Los estudios de pertinencia y factibilidad y el diseño curricular de este nuevo programa de posgrado fueron realizados por la Dra. Carmen Lorenzo Monterrubio, el Dr. Arturo Vergara Hernández, la Mtra. Ma. Esther Pacheco Medina y el Mtro. Gabriel Márquez Ramírez, con la asesoría de la Mtra. Carolina Valero Olmedo, la Mtra. María Teresa Núñez Espino y la Ing. Concepción Vega Gress del Colegio de Posgrado de la UAEH (Lorenzo, 2017).

Después de someter a consideración el resultado de los estudios a las autoridades universitarias, el 13 de septiembre de 2017 la Maestría en Patrimonio Cultural de México fue aprobada por unanimidad por el $\mathrm{H}$. Consejo Universitario de la UAEH.

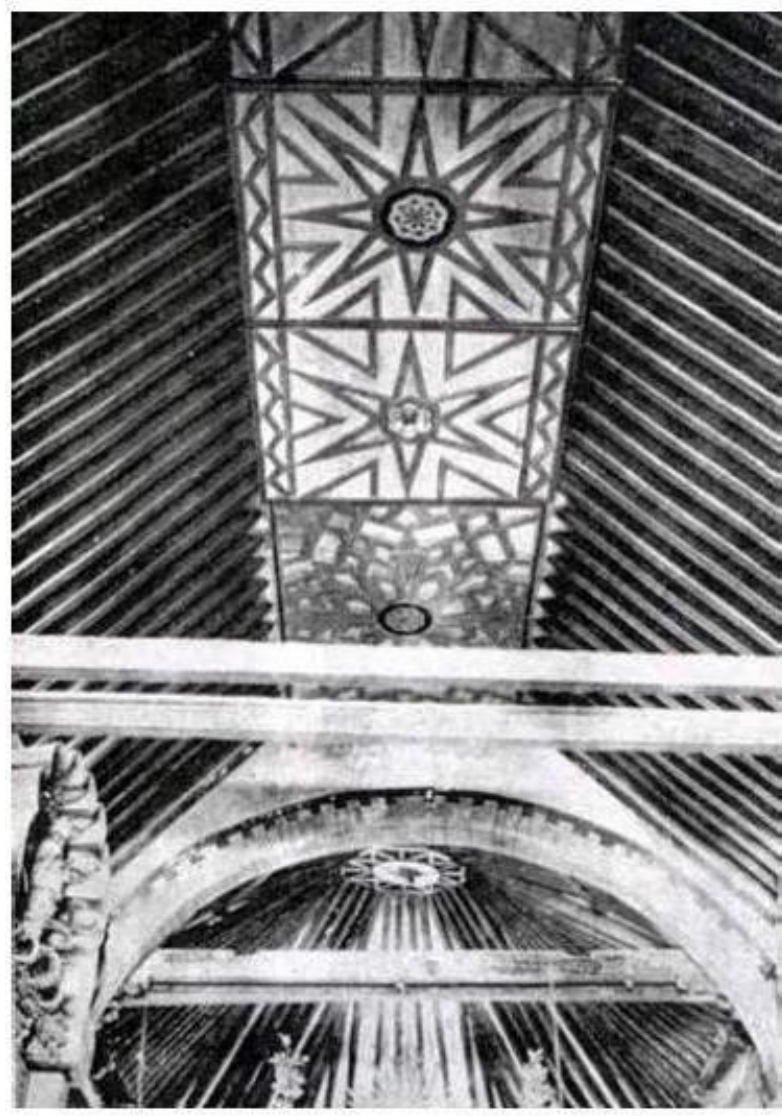

Figura 2. Techo artesonado de la capilla de la Tercera Orden de Tulancingo, demolida en 1962.

Justino Fernández (recopilador). (1984). Catálogo de Construcciones Religiosas del Estado de Hidalgo. Lám. LXXII.

\section{El programa educativo}

Desde un inicio se buscó que la MPCM contribuyera de manera sustancial a la preparación de los profesionistas encargados de la protección y conservación del patrimonio cultural de México y en especial del estado de Hidalgo. Al igual que el resto del país, la entidad hidalguense posee una enorme riqueza patrimonial, tanto en lo material como en lo inmaterial (o vivo) (Arizpe, 2009), por lo que se hace necesario promover y profundizar la profesionalización de esta actividad, que se realiza desde el ámbito civil al público, para lograr el objetivo del programa.

\section{Objetivo}

El objetivo principal de la Maestría en Patrimonio Cultural de México del Instituto de Artes es formar recursos humanos con conocimientos teóricometodológicos, históricos, antropológicos, administrativos, en materia de turismo cultural, desarrollo de empresas e industrias culturales, y 
jurídicas, para intervenir en proyectos con la finalidad de generar una práctica profesional especializada para desempeñarse laboralmente en organismos públicos y privados encargados del patrimonio cultural.

\section{Misión}

La Misión es formar maestros en Patrimonio Cultural de México con una sólida preparación académica, con principios éticos y comprometidos con el estudio multidisciplinar del Patrimonio Cultural, por medio de saberes y competencias que coadyuven a la comprensión, promoción, gestión, protección, conservación y difusión del Patrimonio Cultural de México.

\section{Visión}

En el año 2023, la Maestría en Patrimonio Cultural de México es un programa de excelencia con una alta aceptación en el medio cultural, por la calidad académica de sus egresados con principios éticos y una sólida formación, y por la capacidad de su profesorado que conforman cuerpos académicos consolidados, participan en redes y cultivan Líneas de Generación y Aplicación del Conocimiento, orientadas a la comprensión, promoción, gestión, protección, conservación y difusión del Patrimonio Cultural de México.

\section{Diseño curricular}

El programa de posgrado se cursa en dos años (cuatro semestres), con 14 asignaturas que se integran en tres áreas de formación: Metodológica, Disciplinar e Intervención. En la primera se desarrolla el proyecto de intervención (no precisamente de investigación, pues se trata de una maestría profesionalizante) durante todo el programa, con las siguientes asignaturas: Metodología de la Investigación, Planteamiento del Proyecto Terminal, Desarrollo del Proyecto Terminal y Ejecución del Proyecto Terminal. El área Disciplinar contempla las asignaturas: Patrimonio Prehispánico, Patrimonio Novohispano, Patrimonio de los siglos XIX y XX, Aspectos Teóricos del Patrimonio Cultural, Marco Legal del Patrimonio Cultural, Gestión del Patrimonio Cultural, Administración Aplicada al Patrimonio Cultural, Turismo y Patrimonio Cultural y una materia optativa. El área de Intervención se lleva a cabo en una Estancia Profesional, entre el segundo y tercer semestre, y una Práctica Profesional en el cuarto semestre.

Las asignaturas optativas son: Patrimonio minero, Patrimonio industrial, Patrimonio intangible,
Patrimonio Urbano Contemporáneo, Políticas Públicas y Patrimonio Cultural y Museología.

Se buscó que el diseño curricular de la maestría fuera flexible, por lo que ninguna asignatura es seriada. La posibilidad de extender el proceso de enseñanzaaprendizaje del aula a un escenario real se planteó, desde el diseño del programa, en cuatro salidas de campo al semestre, para que el alumno estuviera en contacto directo con las problemáticas relacionadas con el patrimonio cultural y con la posibilidad de ofrecer alternativas de solución.

Otras actividades académicas relacionadas con el programa son los coloquios internos, donde al final de semestre los alumnos presentan los avances de sus proyectos de intervención, además de conferencias magistrales con especialistas invitados, simposios (como el Primer Encuentro de Investigación sobre Muralismo en Hidalgo, realizado el pasado 6 de noviembre de 2018) y cursos-talleres.

\section{Mapa curricular}

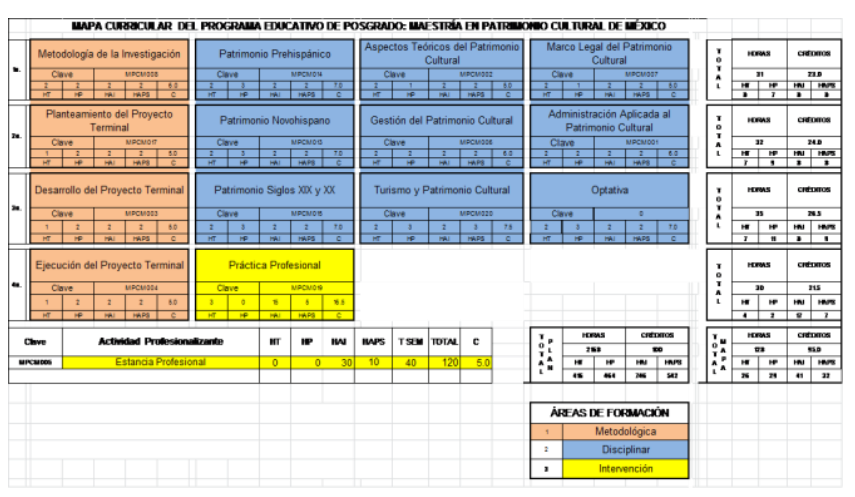

Figura 3. Mapa curricular

\section{Apertura}

\section{La primera generación}

En julio de 2018 ingresó la primera generación de alumnos de la maestría. De 66 aspirantes iniciales se aceptaron solo a 20, los que obtuvieron los mejores puntajes en el examen de admisión. De ellos seis son historiadores, tres licenciados en turismo, dos en arquitectura, dos de teatro, uno en antropología social, uno en gastronomía, uno en diseño gráfico, uno en ciencias de la comunicación, uno en medios masivos de comunicación y uno en administración. Es probable que ningún programa de posgrado cuente con tal diversidad de perfiles previos de licenciatura, que hacen de este programa único en su tipo a nivel nacional, pues 
también en su planta de profesores, confluyen diversas disciplinas humanísticas para el estudio de un solo objeto de estudio. Esto otorga al proyecto interesantes características, retos y oportunidades.

\section{Retos}

El estudio del Patrimonio Cultural desde la maestría que se propone implica muchos retos. Se deben buscar por ejemplo nuevos instrumentos conceptuales y metodológicos para analizar las interacciones entre lo popular y lo masivo, lo tradicional y lo moderno. Es necesario analizar y en su caso proponer modificaciones a la Ley Federal sobre Monumentos y Zonas Arqueológicas, Artísticas e Históricas para adaptarla a los nuevos tiempos globales. Es necesario también revisar los aspectos normativos, legales $y$ administrativos relacionados con todo aquello que se considera patrimonio, especialmente lo relativo al uso y usufructo del patrimonio cultural.

Es necesario, de acuerdo con Enrique Florescano (Pérez, 2018), analizar y solucionar el conflicto entre la defensa del patrimonio arqueológico y la degradación de los sitios en relación con el turismo, buscando el equilibrio entre la conservación y modernización del mismo.

Debe detenerse el enfoque tradicional que busca construir una cultura nacional sustentada en la destrucción de las culturas particulares y en la imposición de sus propios valores.

Según Guillermo Bonfil (2003), la cultura y el patrimonio nacional deben ser un campo de diálogo y de intercambio de experiencias y conocimientos y reconocimientos mutuos, es decir, pluricultural.

Es necesario identificar y dignificar los sistemas de pensamiento y valores mesoamericanos que han sido sistemáticamente desdibujados desde hace 500 años.

\section{Ingreso al PNPC del CONACyT}

Una aspiración del programa de posgrado es ingresar al Programa Nacional de Posgrados de Calidad (PNPC) del Consejo Nacional de Ciencia y Tecnología (CONACyT) pues además del prestigio que esto conlleva, la posibilidad de becar a los estudiantes mejorará notablemente el perfil de egreso. Lograr la calidad académica es una prioridad de la maestría, por lo que se plantea la necesidad de contar con profesores especialistas altamente capacitados y reconocidos en el ámbito de su profesión. Cada una de las asignaturas del programa es un reto para el Instituto de Artes, ya que son pocos los profesores que se dedican a cada una de las diferentes áreas relacionadas con el patrimonio cultural de México, y sobre todo del estado de Hidalgo.

Es importante reconocer las características específicas de este programa de posgrado, tanto en el diseño curricular como en los procesos de enseñanzaaprendizaje. Nuevas y mejores tecnologías son ahora necesarias para la comprensión, difusión y protección del patrimonio cultural (diseños multimedia, imágenes en 3D, propuestas interdisciplinarias y multidisciplinarias, entre otras). La actualización tecnológica va de la mano a la propuesta de proyectos innovadores y novedosos, sin dejar a un lado la identidad comunitaria de los pueblos, su cosmovisión, el respeto a los recursos naturales, la sostenibilidad del patrimonio cultural y la incidencia de política públicas tendientes a la protección de los bienes culturales.

\section{Futuras generaciones}

Las generaciones actuales tienen la obligación de respetar y conservar el patrimonio cultural de México para el disfrute de las venideras, y que éstas continúen esta salvaguarda. Ya no podemos ser indiferentes a la pérdida gradual de la gráfica rupestre, sitios arqueológicos, monumentos históricos, fiestas tradicionales, ceremonias religiosas, artesanías, memoria histórica, lenguas, tradiciones, costumbres y un largo etcétera. Además debemos asumir de manera responsable y sustentable su dimensión económica y su potencial contribución a la mejora de los indicadores económicos.

Los vínculos interinstitucionales son esenciales para lograr una eficaz intervención de protección y conservación del patrimonio cultural. Ahora son más las instituciones educativas y culturales, tanto nacionales como internacionales, que se dedican o se preocupan por el patrimonio cultural. No debemos olvidar que de acuerdo con García Canclini (2018), el patrimonio cultural se debe estudiar no solo como espacio de unidad, sino de lucha material y simbólica entre clases, etnias y grupos sociales. Es necesario conciliar los intereses de los tres principales protagonistas: el Estado, el sector privado y los movimientos sociales.

\section{Conclusiones}

En la situación que ahora vivimos, de globalización, era necesario abrir en el estado de Hidalgo un programa de posgrado dedicado al patrimonio cultural, que en muchos casos es tan débil y frágil que se pierde irremediablemente, y una vez perdido ya no se puede recuperar. Es así como se han acabado lenguas, pueblos originarios, ideologías, cultura material, formas 
de vida y organizaciones comunitarias. Como ejemplos de esta pérdida podemos mencionar la desaparición de algunas tradiciones de las que solo queda algún registro, como la que existió en Tulancingo, de colocar a la entrada de las casas, los días 24 de junio, una ofrenda en honor de San Juan Bautista, en la que debía estar presente el agua y en la que se ponía de manifiesto el ingenio de los habitantes de esta población; otro muy lamentable es la disminución de la lengua tepehua en nuestro estado, pues quedan muy pocos hablantes en la zona de Huehuetla. Otro sería la pérdida de antiguas casonas, portales y atrios de muchas iglesias, que han desaparecido en aras de un mal entendido progreso. Una maestría dedicada a la profesionalización de especialistas encargados de la salvaguarda del patrimonio cultural se hace entonces urgente.

No queda más que desear una larga vida a este nuevo programa posgrado, debido a que existe un interés cada vez mayor de la sociedad por comprender, investigar, promover, difundir, gestionar, proteger, conservar y por qué no decirlo, vivir de nuestro patrimonio cultural.

\section{Referencias}

Arizpe, Lourdes. (2009). El Patrimonio Cultural inmaterial de México, ritos y festividades. México: Consejo Nacional para la Cultura y las Artes, Universidad Nacional Autónoma de México, Miguel Ángel Porrúa.

Bonfil Batalla, Guillermo. (2003). "Nuestro patrimonio cultural: un laberinto de significados" en Patrimonio Cultural y Turismo. Cuadernos. 3 pensamiento acerca del Patrimonio Cultural. Antología de textos. México: Consejo Nacional para la Cultura y las Artes. pp. 45-70.

Fernández, Justino (recopilador). (1984, 1ª ed. 1942). Catálogo de Construcciones Religiosas del Estado de Hidalgo. Vol. II. México: Gobierno del Estado de Hidalgo.

Florescano, Enrique (compilador). (1993). El patrimonio cultural de México. México: Fondo de Cultura Económica, Consejo Nacional para la Cultura y las Artes.

Fosado Martínez. Dulce Olivia y Silvia Nicolás Cisneros. (2011). Estudios de Pertinencia y Factibilidad. Maestría en Historia del Arte. México: Universidad Autónoma del Estado de Hidalgo.
García Canclini, Néstor. "Los usos sociales del Patrimonio Cultural", en Cuadernos del Departamento de Antropología de la UAM Iztapalapa. Recuperado

https://www.iaph.es/export/sites/default/galerias/documentacion_migra cion/Cuaderno/1233838647815_ph10.nestor_garcia_canclini.capii.pdf, el 7 de diciembre de 2018.

Lorenzo Monterrrubio, Carmen (responsable). (2017). Programa educativo de la Maestría en Patrimonio Cultural de México. México: Universidad Autónoma del Estado de Hidalgo.

Pérez Ruiz, Maya Lorena. Reseña de "El patrimonio cultural de México" de Enrique Florescano. (comp.). Alteridades [en línea] 1998, 8 (juliodiciembre). Recuperado de: http://www.redalyc.org/articulo.oa?id=74781619, el 5 de diciembre de 2018. 